\title{
LPR-MLP: A Novel Health Prediction Model for Transmission Lines in Grid Sensor Networks
}

\author{
Yunliang Chen $\mathbb{D}^{1},{ }^{1,2}$ Shaoqian Chen $\mathbb{D}^{1,3}$ Nian Zhang ${ }^{1}{ }^{1}$ Hao Liu ${ }^{1}{ }^{1}$, Honglei Jing $\left(\mathbb{D},{ }^{1}\right.$ \\ and Geyong Min (iD) \\ ${ }^{1}$ School of Computer Science, China University of Geosciences, Wuhan 430074, China \\ ${ }^{2}$ Hubei Key Laboratory of Intelligent Geo-Information Processing, China University of Geosciences, Wuhan 430074, China \\ ${ }^{3}$ School of Economics and Management, China University of Geosciences, Wuhan 430074, China \\ ${ }^{4}$ College of Engineering, Mathematics, and Physical Sciences, University of Exeter, EX4 4QF, Exeter, UK
}

Correspondence should be addressed to Nian Zhang; tozhangnian@gmail.com

Received 18 September 2020; Revised 26 October 2020; Accepted 21 January 2021; Published 9 February 2021

Academic Editor: Weitong Chen

Copyright ( $\odot 2021$ Yunliang Chen et al. This is an open access article distributed under the Creative Commons Attribution License, which permits unrestricted use, distribution, and reproduction in any medium, provided the original work is properly cited.

The safety of the transmission lines maintains the stable and efficient operation of the smart grid. Therefore, it is very important and highly desirable to diagnose the health status of transmission lines by developing an efficient prediction model in the grid sensor network. However, the traditional methods have limitations caused by the characteristics of high dimensions, multimodality, nonlinearity, and heterogeneity of the data collected by sensors. In this paper, a novel model called LPR-MLP is proposed to predict the health status of the power grid sensor network. The LPR-MLP model consists of two parts: (1) local binary pattern (LBP), principal component analysis (PCA), and ReliefF are used to process image data and meteorological and mechanical data and (2) the multilayer perceptron (MLP) method is then applied to build the prediction model. The results obtained from extensive experiments on the real-world data collected from the online system of China Southern Power Grid demonstrate that this new LPR-MLP model can achieve higher prediction accuracy and precision of $86.31 \%$ and $85.3 \%$, compared with four traditional methods.

\section{Introduction}

Smart grid has become a critical factor for the healthy development of smart cities and our social lives. The development of smart grids can be improved by modern technologies such as artificial intelligence and Internet-ofThings for providing smarter and convenient services. As basic setting of the smart grid, the sensor network based monitoring system is applied for detecting the health of the power grid, especially for the transmission lines.

Power stations are usually located in remote areas that often need to have long transmission distance, and, along the distant way, the areas are with harsh geographical environment and complicated micrometeorological conditions. Especially under extreme weather, the ice and wind disasters would cause a large number of grid accidents $[1,2]$. In the spring of 2008, a rare ice and wind disaster spread in southern China, forcing 7541 transmission lines above $10 \mathrm{kV}$ to be shut down, thousands of poles and towers to be inverted, and more than 2000 lines to be disconnected $[3,4]$. Therefore, it is important and urgent to evaluate the health level of transmission lines and make countermeasures so as to prevent the occurrence of freezing disaster [5].

The health of transmission lines is affected by many factors, among which the icing is the critical one. When transmission lines are overly iced, it will break due to their own gravity and certain wind force and even pull down the tower pole. Research on ice-covered loads has been conducted for decades, and many results have been obtained. The icing of the wire is related to many meteorological data. In general, it is the result of the combined action of two mechanisms, thermodynamics and fluid mechanics [6]. Although the icing of transmission lines in the power grid is one of the main causes of grid failures, more factors such as 
various meteorology, mechanics, and material properties also should be considered in evaluating the health status of transmission lines.

As a tool for human beings to understand and describe the world and various phenomena, the mathematics and physics models based on mathematical tools provide the theoretical models which can best measure the health status of transmission lines. A lot of work has been carried out and achieved several positive results [7-9]. A modified Firework Algorithm (FA) based on time series [10] is established for transmission lines to guide the prediction of the current health status of transmission lines. A heuristic transmission line fault model based on microclimate analysis provides a reference for regional transmission line fault prediction [11]. A quantitative model for meteorological factors of transmission line failure rate based on exponential function was proposed [12], which effectively explains some factors that were discovered and explained for line failure before. An exponential function based model [12] was proposed to quantify the weather factor that is relevant to the transmission line failure rate, effectively explaining some implicit factors. However, in practical applications, mathematical models need clear and standardized parameters, which are not suitable for solving nonlinear, complex, diverse, and high-dimensional problems $[13,14]$. As a popular research field in machine learning, the neural network uses a combination of simple functions to approximate complex functions, and its single-hidden layer neural network can approach a large class of functions on a compact subset [15], which has outstanding performance in the modeling of complex data sets.

However, in the scenario of monitoring or predicting the health of transmission lines, the data, which is collected through sensors network, including image data, meteorological data, and mechanical data, are highly dimensional, nonlinear, multimodal, and heterogeneous. These data features lead to not only a low accuracy of the abovementioned models but also a high cost of training these models. In addition, all these algorithms only considered the single particular category of monitoring data but did not consider image data. Therefore, establishing a high-performance model for the health prediction of transmission lines has become a key problem to be addressed urgently.

To cope with this challenge, this paper proposes a novel hybrid model called LPR-MLP to predict the health level of transmission lines. LPR-MLP can efficiently analyze structured data and unstructured image data altogether. Firstly, the features of image data, meteorological data, and mechanical data are extracted by using local binary patterns (LBP) [16], principal component analysis (PCA) [17, 18], and ReliefF [19]. Then, the multilayer perceptron (MLP) with one hidden layer is applied to form the prediction model for the health level of transmission lines. The data monitored by the China Southern Power Grid Online Monitoring System from 2013 to 2016 were collected to evaluate the performance of our proposed prediction model. By comparing with the existing random forest (RF) model, Naive Bayes (NB) model, support vector machine (SVM) model, and decision tree (DTree) model, the results show that the proposed hybrid LPR-MLP approach demonstrates the best performance in terms of accuracy, recall, and precision.

The rest of this paper is organized as follows: in section 2, we introduce and discuss the related work. In section 3 , we develop the LPR-MLP algorithm for the health level prediction model. The detailed experimental evaluation is illustrated using different metrics in Section 4. Section 5 concludes this work and provides the future direction of this work.

\section{Related Work and Preliminary}

2.1. Image Texture Feature Extraction. Image texture feature is a global feature, which describes the surface properties of the scene for the image or the image's area. Since image texture has the characteristics of universality and diversity, there is not a standard definition of image texture. One widely accepted definition is that texture is a visual feature that reflects homogeneous phenomena in an image and embodies the inherent attributes common to the object's surface, including the important information of the structure and arrangement of the object surface and their relationship with the surrounding environment [20,21]. Texture feature extraction is the key link for successful image texture description, classification, and segmentation. The targets of the extraction are as follows: the extracted texture features have small dimensions, strong discriminating ability, good robustness, and a small amount of calculation during the extraction process and can guide practical applications [22]. As a statistical feature, texture features often have rotation invariance and strong resistance to noise.

There are many methods to study image texture features, including statistical analysis method, structural analysis method, model analysis method, and spectrum analysis method [23]. The texture features of pictures have great randomness locally, but there are some rules in the overall and statistical significance, so the statistical analysis method is most widely used to extract texture features. The classical statistical analysis methods are gray level cooccurrence matrix (GLCM) [20] and local binary pattern (LBP) [24]. GLCM is a common method to describe texture by studying the spatial correlation of gray level. Based on the estimation of the second-order combined conditional probability density of the image, GLCM describes the joint distribution of gray level of two pixels with a certain spatial position relationship. LBP is an algorithm used to extract the local texture features of an image. Based on the auxiliary measurement of the local contrast of the image, it extracts the local texture features of the image. It has significant advantages such as rotation invariance and gray invariance. Its feature discrimination power and low computational complexity make it widely used in the fields of image analysis, computer vision, and pattern recognition.

2.2. Data Dimensionality Reduction Technology. With the advent of the era of big data, massive data has grown exponentially, and some researchers have proposed the 
concept of "Curse of Dimensionality" [25]. How to find the segment and extract the data needed by researchers from massive data has always been a hot issue for the majority of scientific researchers. Data dimensionality reduction is one of the key technologies to solve this problem. PCA is a classic data dimensionality reduction algorithm.

Principal component analysis (PCA), proposed by Pearson [17] in 1901 and developed by Hotelling [18], is a widely used data dimensionality reduction algorithm. PCA is a linear dimensionality reduction method that transforms input data through linear mapping relationships. It can be used for both classification and regression problems. The main idea is to map $n$-dimensional features to $k$-dimensional $(k<n)$. This $k$-dimension is a new orthogonal feature, which is called the main component.

The core idea of PCA is to map the data of high-dimensional space to low dimensional space through some linear projection and expect that the variance of data in the projected dimension is the largest. For a data set $X=\left\{x_{1}, x_{2}, \ldots, x_{m}\right\}$ of a high-dimensional space $R^{n}$, the dimension of each data $x_{i}(i=1,2, \ldots, m)$ is $n$, namely, $X \in R^{n \times m}$. Through mapping, we can get the data set $Y=\left\{y_{1}, y_{2}, \ldots, y_{m}\right\} \in R^{d \times m}$, and the objective function of the PCA method is as follows:

$$
\varphi_{\mathrm{PCA}}=\arg \max \sum_{i=1}^{m} y_{i}-\frac{1}{m} \sum y_{i}^{2} .
$$

Due to $y_{i}=W^{T} x_{i}$, equation (1) can be written as follows:

$$
\begin{aligned}
\varphi_{\mathrm{PCA}} & =\arg \max \sum_{i=1}^{m}\left\|W^{T} x_{i}-\frac{1}{m} \sum W^{T} x_{i}^{2}\right\|^{2} \\
& =\arg \max \sum_{i=1}^{m}\left\|W^{T}\left(x_{i}-\frac{1}{m} \sum x_{i}\right)\right\|^{2} \\
& \left.\left.=\arg \max \sum_{i=1}^{m} W^{T}\left(x_{i}-\frac{1}{m} \sum x_{i}\right)\left(x_{i}-\frac{1}{m} \sum x_{i}\right)\right)\right)^{T} W .
\end{aligned}
$$

Simplifying equation (2), we can get

$$
\varphi_{\mathrm{PCA}}=\arg \max \sum_{i=1}^{m} W^{T} C W,
$$

where

$$
\begin{aligned}
C & \left.=\left(x_{i}-\frac{1}{m} \sum x_{i}\right)\left(x_{i}-\frac{1}{m} \sum x_{i}\right)\right)^{T}, \\
W & =\left(\omega_{1}, \omega_{2}, \ldots, \omega_{k}\right), \\
\text { s.t. } y_{i} & =W^{T} x_{i}, W^{T} W=1 .
\end{aligned}
$$

2.3. Artificial Neural Network. As one of the most popular machine learning technologies, artificial neural networks are widely used in various fields and are the fundamental technology of deep learning. In 1943, McCulloch and Pitts [26] proposed the first artificial neuron model and abstracted the mathematical model of the neuron "M-P Neuron Pattern." In 1958, Rosenblatt [27] added a learning mechanism to the original MP model and proposed a perceptron model. The perceptron was composed of two layers of neurons and was able to implement logical AND, OR, and NOT operations, which was a huge improvement at the time, but, unfortunately, it could not handle linear inseparability. McClelland and Rumelhart [28] analyzed the error backpropagation algorithm of multilayer feedforward network, namely, BP algorithm, and put forward a complete $\mathrm{BP}$ algorithm through systematic analysis. BP algorithm has rigorous mathematical derivation, which provides a theoretical basis for neural network weight adjustment. The proposed BP algorithm promoted the rapid development of neural networks and became a milestone in the history of neural network development.

Here, a single-hidden layer feedforward neural network based on the M-P mode and BP algorithm is briefly introduced. A typical single-hidden layer feedforward neural network consists of an input layer, a hidden layer, and an output layer. Let the size of the input layer be $n$, the size of the hidden layer be $h$, and the size of the output layer be $m$. There is any data set $(X, T)=\left\{\left(X_{i}, T_{i}\right) \mid i=1,2, \ldots, N\right\}$, where $X_{i}=\left[X_{i 1}, X_{i 2}, \ldots, X_{i n}\right]^{T}, \quad t_{i}=\left[T_{i 1}, T_{i 2}, \ldots, T_{i m}\right]^{T}$, the overall network of the single-hidden layer can be expressed as

$$
\begin{aligned}
& o_{j}=\left[\begin{array}{c}
o_{1 j} \\
o_{2 j} \\
\vdots \\
o_{m j}
\end{array}\right]=\left[\begin{array}{c}
\sum_{i=1}^{h} \beta_{i 1} g\left(W_{i} X_{j}+b_{i}\right) \\
\sum_{i=1}^{h} \beta_{i 2} g\left(W_{i} X_{j}+b_{i}\right) \\
\vdots \\
\sum_{i=1}^{h} \beta_{i m} g\left(W_{i} X_{j}+b_{i}\right)
\end{array}\right], \quad j=1,2, \ldots, N, \\
& \text { s.t.arg } \min _{T} \sum_{j=1}^{N}\left\|o_{j}-T_{j}\right\|,
\end{aligned}
$$

$\beta_{i}$ is the weight of the hidden layer to the output layer, $W_{i}$ is the weight of the input layer to the hidden layer, $b_{i}$ is the bias of the hidden layer, $g(x)$ is the activation function, and $o_{j}$ is the output of the output layer.

\section{Hybrid Prediction Model}

A new hybrid model LPR-MLP with the joint use of LBP, PCA, ReliefF, and MLP is proposed to predict the health status of transmission lines. LPR is the abbreviation of LBP, PCA, and ReliefF. The LPR-MLP model mainly includes two stages: data preprocessing and model training. The data preprocessing is divided into two parts: one is the texture features extraction for pictures by LBP and PCA, and the other is the dimension reduction for the meteorological and mechanical data by ReliefF. The overall structure of the model is shown in Figure 1. 


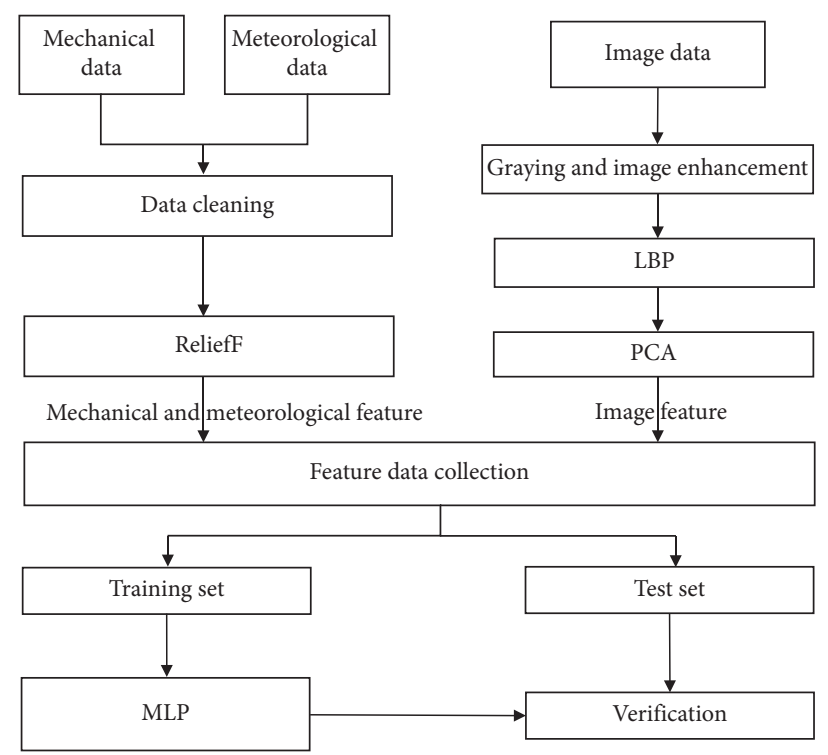

Figure 1: The flowchart of the LPR-MLP model.

3.1. Image Data Preprocessing. The image data comes from the image sensor of the online monitoring system of China Southern Power Grid Corporation. The original image is colored and belongs to the RGB color space. In order to facilitate the subsequent processing, the image is grayed first:

$$
Y=0.299 R+0.587 G+0.114 B,
$$

where $R, G$, and $B$ are the red component value, green component value, and blue component value of the image, respectively. $Y$ is the brightness, that is, the corresponding gray value after the RGB image is converted into a gray image.

In order to enhance the grayscale image contrast and eliminate possible interference and noise in the image, it is necessary to perform an image enhancement operation. First, the gray value of the image is mapped. Set the gray value set of image $P$ pixel as $\left\{X_{i, j} \mid(i, j) \in P\right\}$ :

$$
\begin{aligned}
P_{\text {max }} & =\max \left\{X_{i, j} \mid(i, j) \varepsilon P\right\}, \\
P_{\text {min }} & =\min \left\{X_{i, j} \mid(i, j) \varepsilon P\right\}, \\
Y_{i, j} & =\frac{X_{i, j}-P_{\text {min }}}{P_{\text {max }}-P_{\text {min }}} \times 255 .
\end{aligned}
$$

We define $Y_{i, j}$ to be the gray value after mapping. The maximum gray value in the image is mapped to 255 , the minimum gray value is mapped to 0 , and the remaining gray values are scaled equally. The image used in this paper has the characteristics of low resolution. In order to deal with noise points while retaining more image information, median filtering is used to process image data. The edge pixels in the image are output directly without median filtering. The median filter window size used in this manuscript is $W=9$ :

$$
Z_{i, j}=\operatorname{median}\left\{Y_{i+k, j+l} \mid(k, l) \in W\right\}
$$

where median $\{X\}$ means to take the median of series $X$ and then use the median $Z_{i, j}$ of all gray values in window $W$ as the output of the median filter.

Use the LBP algorithm to extract the texture features of the image and get 531-dimensional feature data. The LBP value of any point $C\left(x_{c}, y_{c}\right)$ is

$$
\begin{aligned}
& \operatorname{LBP}_{N, R}=\sum_{i=1}^{N} 2^{i} T\left(p_{i}-p_{c}\right), \\
& \operatorname{LBP}_{C\left(x_{c}, y_{c}\right)}=\min \left\{\operatorname{ROT}\left(\operatorname{LBP}_{N, R}, i\right) \mid, \quad i=1,2, \ldots, N\right\},
\end{aligned}
$$

where $p_{i}$ represents the gray value of point $i\left(x_{i}, y_{i}\right)$ and $p_{c}$ represents the gray value of point $C$. $R$ is the radius of the sampling circle and $N$ is the number of sampling points. $\operatorname{ROT}(x, i)$ is a rotation function, which means that $x$ is shifted right by $i(i \leq N)$ bits. The function $T(x)$ is defined as

$$
T(x)= \begin{cases}0, & x<0, \\ 1, & x \geq 0 .\end{cases}
$$

The texture is used as the surface property of the scene corresponding to the image or image area. Successful extraction of texture features is helpful for image texture description, classification, and segmentation. However, the texture feature data extracted by LBP has high dimensionality, and some components are even linearly related, which will seriously affect the learning efficiency of the model and the prediction accuracy of the model. PCA is a linear dimensionality reduction method that minimizes the loss of original data information.

For data set $D=\left\{x_{1}, x_{2}, \ldots, x_{m}\right\}$, the dimension of each data $x_{i}(i=1,2, \ldots, m)$ is $n$, which needs to be reduced to $k$ dimension. The specific process is as follows.

Let $X=\left(x_{1}, x_{2}, \ldots, x_{m}\right)$ be a matrix of $n \times m$ : 


$$
X=\left(\begin{array}{c}
x_{1}^{1}, x_{2}^{1}, \ldots, x_{m}^{1} \\
x_{1}^{2}, x_{2}^{2}, \ldots, x_{m}^{2} \\
\ldots \\
x_{1}^{n}, x_{2}^{n}, \ldots, x_{m}^{n}
\end{array}\right)
$$

(1) Centralize all samples:

$$
x_{i}=x_{i}-\frac{1}{m} \sum_{j=1}^{m} x_{j} .
$$

(2) Calculate the covariance matrix $(1 / m) X X^{T}$.
(3) The eigenvalue decomposition method is used to decompose the covariance matrix $(1 / m) X X^{T}$ and get its eigenvalue and eigenvector.

(4) Sort the eigenvalues from large to small, take out the eigenvectors corresponding to the largest $k$ eigenvalues, and then normalize them to form a matrix $W\left(\omega_{1}, \omega_{2}, \ldots, \omega_{k}\right)$.

(5) For each dimension $x_{i}(i=1,2, \ldots, m)$ in the row vector $X$, proceed in order:

$$
y_{i}=W^{T} * x_{i} .
$$

That is, for matrix $X$,

$$
\left(y_{1}, y_{2}, \ldots, y_{m}\right)=\left(\begin{array}{c}
\omega_{1}^{1}, \omega_{1}^{2}, \ldots, \omega_{1}^{n} \\
\omega_{2}^{1}, \omega_{2}^{2}, \ldots, \omega_{2}^{n} \\
\ldots \\
\omega_{k}^{1}, \omega_{k}^{2}, \ldots, \omega_{k}^{n}
\end{array}\right)\left(\begin{array}{c}
x_{1}^{1}, x_{2}^{1}, \ldots, x_{m}^{1} \\
x_{1}^{2}, x_{2}^{2}, \ldots, x_{m}^{2} \\
\ldots \\
x_{1}^{n}, x_{2}^{n}, \ldots, x_{m}^{n}
\end{array}\right)
$$

(6) $Y=W^{T} X$ is the data after dimension reduction to $k$ :

$$
Y=\left(\begin{array}{c}
y_{1}^{1}, y_{2}^{1}, \ldots, y_{m}^{1} \\
y_{1}^{2}, y_{2}^{2}, \ldots, y_{m}^{2} \\
\ldots \\
y_{1}^{k}, y_{2}^{k}, \ldots, y_{m}^{k}
\end{array}\right)=\left(y_{1}, y_{2}, \ldots, y_{m}\right)
$$

3.2. ReliefF Algorithm. For the meteorological and mechanical data obtained by the sensor, there are some missing values and outlier in some one-dimensional features of some data samples. Hence, we need to do data cleaning, fill in null values using the mean filling method, smooth the outlier noise point data, and carry out the dimension reduction using ReleifF [19].

When filling in the null value, the mean of the data from three days before and after is used. If there are still null values in the data of filling data, the value is taken forward (backward) in order. When smoothing outliers, smoothing is performed using the average of the data before and after. After cleaning the data, the ReliefF algorithm is used to reduce the dimensionality of the meteorological and mechanical data in order to find the data features that are strongly related to the transmission line and are not related to each other.

ReliefF algorithm uses "Correlation Statistics" to measure the importance of features, which is a feature weight algorithm. According to the correlation of each feature and category, different weights are given to features, and the feature whose weight is less than the threshold value $\omega_{0}$ will be screened. For data set $D=\left\{x_{1}, x_{2}, \ldots, x_{m}\right\}$, its size is $m$, any data $x_{i}(i=1,2, \ldots, m)$ is composed of $p$-dimensional features, and $z$ represents its category: $x_{i}\left(x_{i}^{1}, x_{i}^{2}, \ldots, x_{i}^{p}, z\right)$. The data set contains a total of $|\gamma|$ categories. For any data $x_{i}$, assume $z=k$; that is, $x_{i}$ is the $k$ th $(k \in\{1,2, \ldots,|\gamma|\})$ class. The ReliefF algorithm first finds the nearest neighbor data $x_{i, H}$ in the samples of class $k$ as the guessing nearest neighbor of $x_{i}$ and then finds the nearest neighbor data $x_{i, t, M}(t=$ $1,2, \ldots,|\gamma| \mid, t \neq k)$ in the samples of each class other than class $k$ as the guessing nearest neighbor of $x_{i}$. Then, the correlation statistic $\delta^{j}$ corresponding to any attribute $j$ in the data sample is

$$
\delta^{j}=\sum_{i}-\operatorname{diff}\left(x_{i}^{j}, x_{i, H}^{j}\right)^{2}+\sum_{t \neq k} p_{t} \times \operatorname{diff}\left(x_{i}^{j}, x_{i, t, M}^{j}\right)^{2},
$$

where $x_{i}^{j}$ represents the value of the data sample $x_{i}$ on attribute $j, p_{t}$ is the proportion of the $\mathrm{t}$-th sample in the data set $D$, and the value of the function diff $(x, y)$ depends on the type of attribute $j$.

(1) When $x$ and $y$ are discrete variables:

$$
\operatorname{diff}(x, y)= \begin{cases}0, & x=y, \\ 1, & \text { otherwise. }\end{cases}
$$

(2) When $x$ and $y$ are continuous variables:

Normalize all values belonging to the same attribute $j$ :

$$
x_{i}^{j}=\frac{x_{i}^{j}-x^{j}{ }_{\text {min }}}{x^{j}{ }_{\max }-x^{j}{ }_{\min }},
$$


where $x_{\max }^{j}$ and $x_{\min }^{j}$ represent the maximum value and the minimum value of all values of the attribute $j$, respectively. After normalizing $x, y$,

$$
\operatorname{diff}(x, y)=|x-y| \text {. }
$$

Then, the weight $W(j)$ of the corresponding attribute $j$ is

$$
W(j)=W(j)+\delta^{j} .
$$

By using ReliefF, the dimension of 12-dimensional meteorological data and 24-dimensional mechanical data are reduced to 6-dimensional and 8-dimensional, respectively.

3.3. Multilayer Perceptrons. Multilayer perceptrons (MLP) are developed from perceptrons [29]. In general, MLP are multilayer feedforward neural networks that use the error backpropagation algorithms $[30,31]$ to learn and update network parameters. In this work, a hidden layer multilayer perceptron is applied for modeling, which is equivalent to a single-hidden layer feedforward neural network (SLFN). A typical SLFN is composed of an input layer, a hidden layer, and an output layer, as shown in Figure 2, where the input layer size is $n$, the hidden layer size is $z$, and the output layer size is $m$. There is a data set $(X, T)=\left\{\left(X_{i}, T_{i}\right) \mid i=1,2, \ldots, N\right\}, \quad$ where $X_{i}=\left[X_{i 1}, X_{i 2}, \ldots, X_{i n}\right]^{T}, T_{i}=\left[T_{i 1}, T_{i 2}, \ldots, T_{i m}\right]^{T}$. It can be obtained that the value $H\left(h_{j}\right)$ of any hidden layer node $h_{j}$ is

$$
H\left(h_{j}\right)=g\left(w_{j} \cdot X_{i}+b_{j}\right), \quad j=1,2, \ldots, z .
$$

Among them, $w_{j} \cdot X_{i}$ represents the inner product between $w_{j}$ and $X_{i} . w_{j}=\left[w_{1 j}, w_{2 j}, \ldots, w_{n j}\right]^{T}$ is the input weight vector of the input layer and the $j$-th hidden layer neuron, $b_{j}$ is the threshold value of the $j$-th hidden layer neuron, and $g(x)$ is the activation function of the hidden layer. According to the value of the hidden layer, the output value $Y_{i}$ corresponding to the output layer can be calculated:

$$
Y_{i}=\sum_{j=1}^{z} \beta_{j} g\left(w_{j} \cdot X_{i}+b_{j}\right), \quad i=1,2, \ldots, N
$$

where $\beta_{j}=\left[\beta_{j 1}, \beta_{j 2}, \ldots, \beta_{j m}\right]^{T}$ is the output weight vector between the $j$-th hidden layer neuron and the output layer. So, the output of the entire neural network is

$$
Y=\left[\begin{array}{llll}
Y_{1} & Y_{2} & \ldots & Y_{N}
\end{array}\right]=\left[\sum_{j=1}^{z} \beta_{j} g\left(w_{j} \cdot X_{1}+b_{j}\right) \sum_{j=1}^{z} \beta_{j} g\left(w_{j} \cdot X_{2}+b_{j}\right): \sum_{j=1}^{z} \beta_{j} g\left(w_{j} \cdot X_{N}+b_{j}\right)\right]^{T} .
$$

\section{Experimental Results}

In this section, we first introduce the generation of the experimental data source and then report the performance of the proposed LPR-MLP model by comparing it with the RF model, NB model, SVM model, and DTree model.

4.1. Data Source. The experimental data in this work was generated from the terminals of the online monitoring system of China Southern Power Grid Corporation, from 2013 to 2016. The data set can be divided into five parts: basic environmental information data, image data, meteorological data, mechanical data, and wire parameters. The image data type is shown in Figure 3. The thickness of icing will affect the health status of transmission lines. Different thickness grades can be calculated according to the model [32], which will reflect the health status of transmission lines to a certain extent. The basic environmental information data includes terminal name and time and date recorded. Meteorological data include temperature, humidity, light intensity, wind speed, and wind. The mechanical data include the maximum and minimum tension, the wind deflection angle under the maximum and minimum tension, and the inclination angle under the maximum and minimum tension. Wire parameters include wire diameter and wire splitting coefficient. The details are shown in Table 1.

Each data sample also has a corresponding health status value, which is obtained by combining with the related mathematical and physical models. It is numerical data with a data range of $[0,1]$ (note: this is not the probability of related transmission line failure, but a value indicating the health status. The higher the value, the worse the health status; otherwise, the better the health status). According to the suggestions of China Southern Power Grid Corporation on the classification of transmission line health level, this paper divides the health status into 7 levels. The corresponding relationship is shown in Table 2.

The data set has a total of 4455 data samples, of which $80 \%$ (a total of 3564 data samples) are used as the training set in the experiments and 20\% (a total of 891 data samples) are used as the test set.

\section{Results and Discussion}

This subsection shows the performance of the proposed model through comparison with other models. Four metrics are used to evaluate the performance of model classification, in terms of accuracy, precision, recall, and MacroF1. 


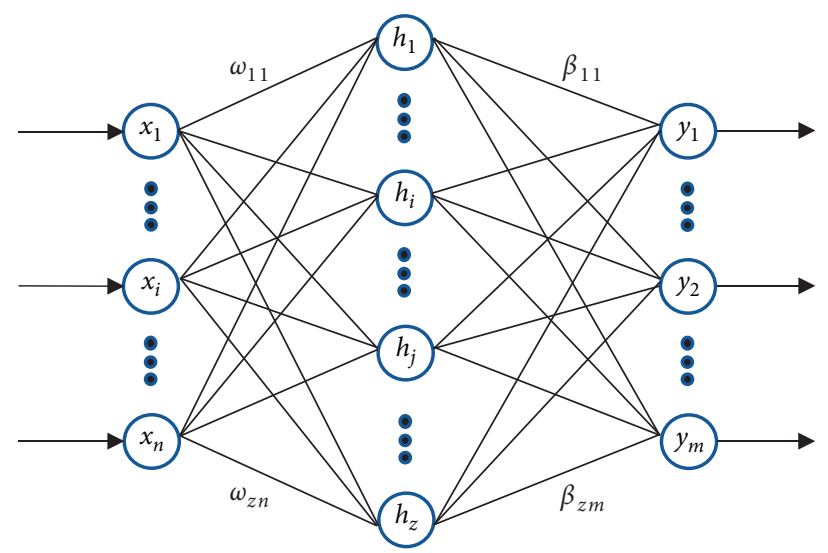

FIgURE 2: Schematic diagram of a single-hidden layer.
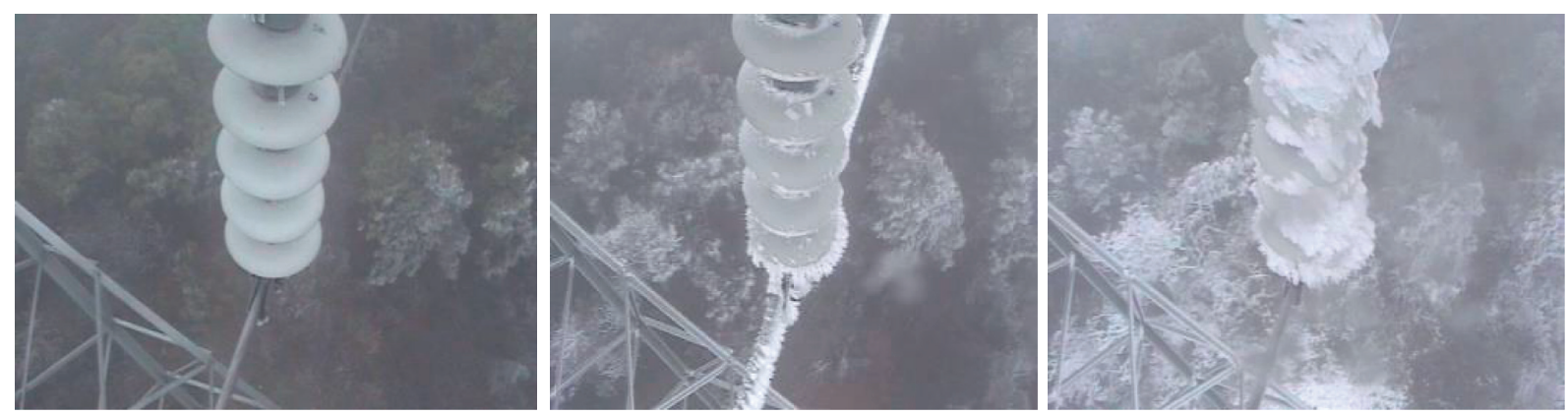

FIgURE 3: Examples of original image data.

TABle 1: Experimental data description.

\begin{tabular}{|c|c|c|}
\hline $\begin{array}{l}\text { Feature } \\
\text { classification }\end{array}$ & Description & $\begin{array}{l}\text { Feature } \\
\text { dimension }\end{array}$ \\
\hline Image data & Process images through local binary mode (LBP) and extract features using histograms & 531 \\
\hline $\begin{array}{l}\text { Meteorological } \\
\text { data }\end{array}$ & Temperature, humidity, light intensity, wind speed, wind direction, etc. & 12 \\
\hline Mechanical data & $\begin{array}{l}\text { Maximum and minimum tension, wind deflection angle at maximum and minimum tension, tilt angle at } \\
\text { maximum and minimum tension, etc. }\end{array}$ & 24 \\
\hline Wire parameters & Wire diameter, wire splitting factor, average equivalent wire length, etc. & 5 \\
\hline
\end{tabular}

TABLE 2: Health classification of transmission lines.

\begin{tabular}{lc}
\hline Health status value & Health levels \\
\hline $0.0-0.1$ & 0 \\
$0.1-0.2$ & 1 \\
$0.2-0.35$ & 2 \\
$0.35-0.5$ & 3 \\
$0.5-0.7$ & 4 \\
$0.7-0.9$ & 5 \\
$0.9-1.0$ & 6 \\
\hline
\end{tabular}

Figure 4 shows the classification accuracy of the LPRMLP, RF model, NB model, SVM model, and DTree model, which are $86.31 \%, 80.92 \%, 62.51 \%, 83.39 \%$, and $80.47 \%$, respectively. The confusion matrix is an important tool for summarizing the prediction results of the classification model. It is necessary to analyze the classification of the confusion matrix in each category. The specific classification of each category in the validation set can be summarized in Table 3 .

In Table 3, the sample size line is the real number from the data source and the categories column indicates the specific classification of each level. The numerator represents the number of correct classification of the level and the denominator represents the number of classification obtained from the prediction model. The whole size of the validation set is 891 . Among them, level 0 has 450 samples, level 1 has 359 samples, level 2 has 32 samples, level 3 has 17 samples, level 4 has 18 samples, level 5 has 10 samples, and level 6 has 5 samples. For each level, the numbers of correct classification of the LPR-MLP model are 366, 337, 25, 15, 14, 9 , and 3 , and the total of these numbers is 769 which is higher than the other methods. In practice, we should pay more attention to the sensitivity of the model at high risk. So, we still focus on a high level such as levels 3, 4, 5, and 6 in our 


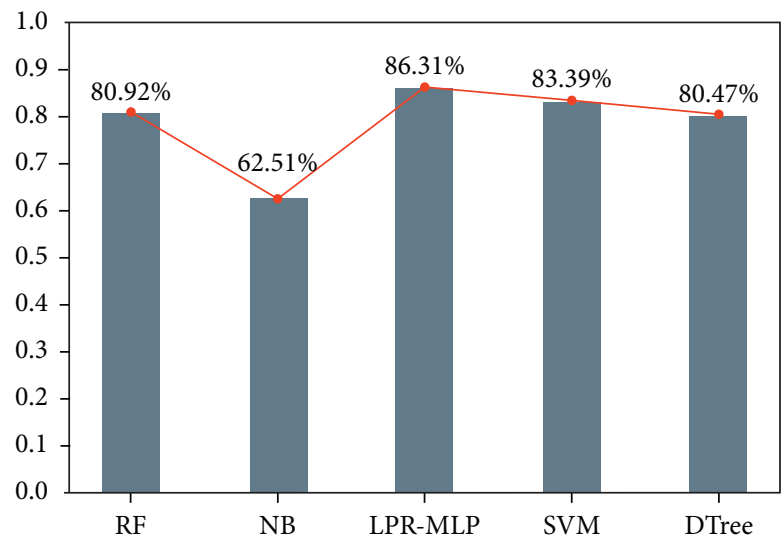

Figure 4: Prediction accuracy of experimental models.

Table 3: Summary of the prediction of each model.

\begin{tabular}{lccccccc}
\hline \multirow{2}{*}{ Models } & & & \multicolumn{3}{c}{ Categories } & & Total \\
& Level 0 & Level 1 & Level 2 & Level 3 & Level 4 & Level 5 & Level 6 \\
\hline RF & $382 / 457$ & $289 / 369$ & $20 / 27$ & $6 / 6$ & $12 / 19$ & $9 / 10$ & $3 / 3$ \\
NB & $436 / 671$ & $78 / 84$ & $25 / 81$ & $7 / 15$ & $7 / 14$ & $0 / 1$ & $4 / 25$ \\
LPR-MLP & $366 / 385$ & $337 / 427$ & $25 / 27$ & $15 / 19$ & $14 / 17$ & $9 / 13$ & $3 / 3$ \\
SVM & $373 / 422$ & $309 / 391$ & $24 / 26$ & $15 / 19$ & $14 / 18$ & $8 / 15$ & 721 \\
DTree & $393 / 483$ & $258 / 318$ & $24 / 40$ & $14 / 17$ & $16 / 18$ & $9 / 12$ & 769 \\
Sample size & 450 & 359 & 32 & 17 & 18 & 10 & 743 \\
\hline
\end{tabular}

TABle 4: Precision of all models.

\begin{tabular}{lccccccc}
\hline Models & & & Categories & & \\
& Level 0 & Level 1 & Level 2 & Level 3 & Level 4 & Level 5 & Level 6 \\
\hline RF & 0.8359 & 0.7832 & 0.7407 & 1 & 0.6316 & 0.9 & 1 \\
NB & 0.6498 & $\mathbf{0 . 9 2 8 6}$ & 0.3086 & 0.4667 & 0.5 & 0 & 0.8416 \\
LPR-MLP & $\mathbf{0 . 9 5 0 6}$ & 0.7892 & $\mathbf{0 . 9 2 5 9}$ & 0.7895 & 0.8235 & 0.6923 & 0.4305 \\
SVM & 0.8839 & 0.7903 & 0.9231 & 0.7895 & 0.7778 & 0.5333 & $\mathbf{1}$ \\
DTree & 0.8136 & 0.8113 & 0.6 & $\mathbf{0 . 8 2 3 5}$ & $\mathbf{0 . 8 8 8 9}$ & $\mathbf{0 . 7 5}$ & 0.6711 \\
\hline
\end{tabular}

system. It also can be calculated that the hit number of the LPR-MLP model at a high level is similar to that of DTree, reaching 41 and 42 while that of other models is 30,18 , and 37. Although the DTree model has a similar rate of high level to the LPR-MLP model, it shows poor accuracy at a low level which cannot be ignored.

In order to illustrate the better performance of the LPRMLP model, Table 4 indicates the precision of the RF model, NB model, LPR-MLP model, SVM model, and DTree model under different health levels. In the CATEGORYS column of the table, each column represents the precision of the five models under these health levels. The last column of the table represents the macroprecision (MacroP) of the five models. It can be concluded that the LPR-MLP model has the highest macroprecision, reaching 0.853 , while the NB model has the lowest macroprecision, only 0.4305 . Specific to each level, the LPR-MLP model has the highest precision on level 0 , level 2 , and level $6(0.9506,0.9259,1.0)$ and acceptable value on level 3 , level 4 , and level $5(0.789,0.823$, and 0.692$)$. The precision of RF models can achieve $84.1 \%$ and have the highest precision on level 3, level 5, and level 6 (1.0, 0.9, and 1.0). However, due to the low accuracy, the RF model cannot be considered yet.

Table 5 shows the recall rate of five models at different health levels. The last column represents the macrorecall (MacroR) of each model. We can see that the macrorecall of the LPR-MLP model is 0.8133 , which is the highest of all models. Specific to each level, the LPR-MLP model has the highest accuracy rate on level 1 , level 2, level 3, and level $5(0.9387,0.7813,0.8824$, and 0.9$)$ and reasonable value on the other levels. Among the high-risk levels from level 4 to level 6, only the DTree model (on level 4) and the NB model (on level 6) have a higher recall rate than the LPR-MLP model. Figure 5 shows the MacroF1 values of the five models. It is obvious that the MacroF1 value of LPR-MLP is the highest. It can reach 0.8211 , while that of the NB, RF, SVM, and DTree model is $0.3877,0.7335$, 0.6797 , and 0.7931 , respectively. 
TABLE 5: Recall of all models.

\begin{tabular}{lccccccc}
\hline Models & & \multicolumn{3}{c}{ Categories } & & \\
& Level 0 & Level 1 & Level 2 & Level 3 & Level 4 & Level 5 & Level 6 \\
\hline RF & 0.8488 & 0.805 & 0.625 & 0.3529 & 0.6667 & 0.9 & 0.6 \\
NB & $\mathbf{0 . 9 6 8 9}$ & 0.2173 & 0.7813 & 0.4118 & 0.3889 & 0 & 0.6855 \\
LPR-MLP & 0.8133 & $\mathbf{0 . 9 3 8 7}$ & $\mathbf{0 . 7 8 1 3}$ & $\mathbf{0 . 8 8 2 4}$ & $\mathbf{0 . 7 7 7 8}$ & $\mathbf{0 . 9}$ & 0.8 \\
SVM & 0.8289 & 0.8607 & 0.75 & 0.8824 & 0.7778 & 0.8 & 0.5097 \\
DTree & 0.8733 & 0.7187 & 0.75 & 0.8235 & 0.8889 & 0.9 & 0.8133 \\
\hline
\end{tabular}

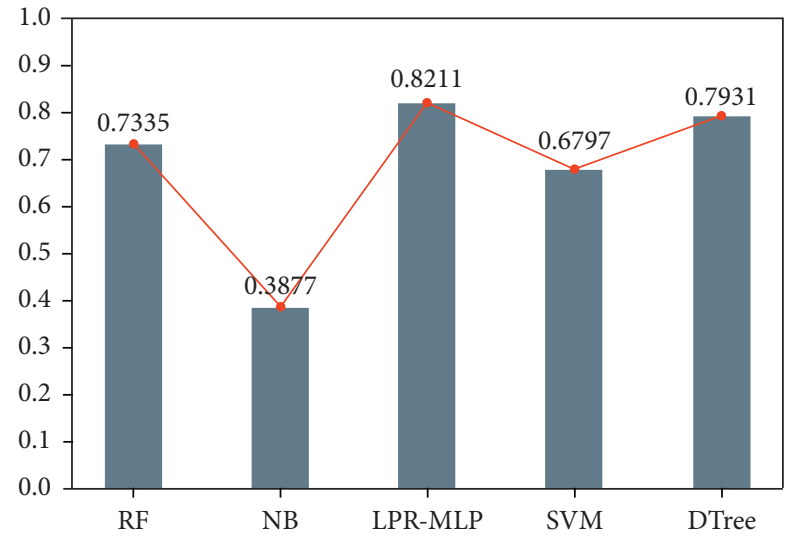

Figure 5: MacroF1 value of the experimental models.

In summary, the proposed LPR-MLP can efficiently raise the prediction accuracy. The results have validated the feasibility and efficiency of the proposed hybrid heath prediction method.

\section{Conclusions and Future Work}

In order to ensure the effective operation of the city's economy and society, it is necessary to accurately evaluate the health level of transmission lines in the power grid sensor network and master the health status of transmission lines in real time. This paper proposes a new LPR-MLP hybrid model, which uses LBP, PCA, and ReliefF to process image data and meteorological mechanics data, respectively, and then uses MLP to predict its health level, thus solving the challenge of predicting the health status of transmission lines under high-dimension, multimode, nonlinear, and heterogeneous data. The experimental results have shown that the LPR-MLP model has higher prediction accuracy and better performance than the other four classical prediction models.

The LPR-MLP model proposed in this paper provides a new idea and effective methods for the health prediction of transmission lines, but the disadvantage is that the feature extraction of image data is a bit rough. In the future, we will consider more scientific methods for processing image data. For example, we may use deep learning technology to process image data in order to get more effective features. As for the meteorological and mechanical data, we can pay more attention to the physical connotation of the data in the future and consider its practical significance before extracting the characteristics.

\section{Data Availability}

The data used to support the findings of this study are available from the corresponding author upon request.

\section{Conflicts of Interest}

The authors declare that there are no conflicts of interest regarding the publication of this article.

\section{Acknowledgments}

This paper was partially supported by the National Natural Science Foundation of China (nos. U1711266, 62076224, and 41925007).

\section{References}

[1] E. L. Lecomte, A. W. Pang, and J. W. Russell, Ice Storm'98, Institute for Catastrophic Loss Reduction Ottawa, Canada, 1998.

[2] L. Wang, Q. Sun, and J.-q. Liu, "Comparative analysis on measures taken by China and other countries to cope with power grid blackouts caused by ice storms," Electric Power Technologic Economics, vol. 2, 2008.

[3] D. Shao, X. Yin, Q. Chen et al., "Affects of icing and snow disaster occurred in 2008 on power grids in South China," Power System Technology, vol. 33, no. 5, pp. 38-43, 2009.

[4] D.-c. Xu, Y.-c. Huang, X.-l. Bai, and P.-f. Han, "Causes of transmission line icing damages and how to improve the design according to new national standard," Power System and Clean Energy, vol. 1, 2011.

[5] J. Liu, Y. Xue, K. Ren, J. Song, C. Windmill, and P. Merritt, "High-performance time-series quantitative retrieval from satellite images on a GPU cluster," IEEE Journal of Selected Topics in Applied Earth Observations and Remote Sensing, vol. 12, no. 8, pp. 2810-2821, 2019.

[6] T. W. Cease and P. Johnston, "A magneto-optic current transducer," IEEE Transactions on Power Delivery, vol. 5, no. 2, pp. 548-555, 1990.

[7] J. Lei and Q. Gong, "Comprehensive prediction method for failure rate of transmission line based on multi-dimensional cloud model," IET Generation, Transmission \& Distribution, vol. 13, no. 9, pp. 1672-1678, 2019.

[8] J. Wang, X. Xiong, N. Zhou, Z. Li, and W. Wang, "Early warning method for transmission line galloping based on SVM and AdaBoost bi-level classifiers," IET Generation, Transmission \& Distribution, vol. 10, no. 14, pp. 3499-3507, 2016.

[9] C. Algora, R. Peña, L. Jiang, J. Liu, G. Zhu, and S. Dai, "Recharging the battery of implantable biomedical devices by light," Artificial Organs, vol. 33, no. 10, pp. 855-860, 2009. 
[10] X. B. Huang, H. B. Li, and Y. C. Zhu, "Short-term ice accretion forecasting model for transmission lines with modified timeseries analysis by fireworks algorithm," IET Generation, Transmission \& Distribution, vol. 12, no. 5, pp. 1074-1080, 2018.

[11] W. Gao, R. Zhou, and D. Zhao, "Heuristic failure prediction model of transmission line under natural disasters," IET Generation, Transmission \& Distribution, Transmission \& Distribution, vol. 11, no. 4, pp. 935-942, 2017.

[12] Y. Wang, L. Chen, M. Yao, and X. Li, "Evaluating weather influences on transmission line failure rate based on scarce fault records via a bi-layer clustering technique," IET Generation, Transmission \& Distribution, vol. 13, no. 23, pp. 5305-5312, 2019.

[13] J. Li, C. Liu, J. X. Yu, Y. Chen, T. Sellis, and J. S. Culpepper, "Personalized influential topic search via social network summarization," IEEE Transactions on Knowledge and Data Engineering, vol. 28, no. 7, pp. 1820-1834, 2016.

[14] J. Han, A. Jentzen, and E. Weinan, "Solving high-dimensional partial differential equations using deep learning," Proceedings of the National Academy of Sciences, vol. 115, no. 7-9, 2017.

[15] A. Pinkus, "Approximation theory of the MLP model in neural networks," Acta Numerica, vol. 8, pp. 143-195, 1999.

[16] T. Ojala, M. Pietikainen, and T. Maenpaa, "Multiresolution gray-scale and rotation invariant texture classification with local binary patterns," IEEE Transactions on Pattern Analysis and Machine Intelligence, vol. 24, no. 7, pp. 971-987, 2002.

[17] K. Pearson, "LIII. On lines and planes of closest fit to systems of points in space," The London, Edinburgh, and Dublin Philosophical Magazine and Journal of Science, vol. 2, no. 11, pp. 559-572, 1901.

[18] H. Hotelling, "Analysis of a complex of statistical variables into principal components," Journal of Educational Psychology, vol. 24, no. 6, p. 417, 1933.

[19] I. Kononenko, "Estimating attributes: analysis and extensions of RELIEF," Machine Learning: ECML-94, vol. 784, pp. 171$182,2005$.

[20] R. M. Haralick, K. Shanmugam, and I. H. Dinstein, "Textural features for image classification," IEEE Transactions on Systems, Man, and Cybernetics, vol. SMC-3, no. 6, pp. 610-621, 1973.

[21] R. M. Haralick, "Statistical and structural approaches to texture," Proceedings of the IEEE, vol. 67, no. 5, pp. 786-804, 1979.

[22] L. Liu and G. Kuang, "Overview of image textural feature extraction methods," Journal of Image and Graphics, vol. 14, no. 4, pp. 622-635, 2009.

[23] J.-D. Sun and Y.-Y. Ma, "Summary of texture feature research," Computer Systems \& Applications, vol. 6, 2010.

[24] T. Ojala, M. Pietikäinen, and D. Harwood, "A comparative study of texture measures with classification based on featured distributions," Pattern Recognition, vol. 29, no. 1, pp. 51-59, 1996.

[25] R. E. Bellman, Adaptive Control Processes: A Guided Tour, Princeton University Press, Princeton, NJ, USA, 2015.

[26] W. S. McCulloch and W. Pitts, "A logical calculus of the ideas immanent in nervous activity," The Bulletin of Mathematical Biophysics, vol. 5, no. 4, pp. 115-133, 1943.

[27] F. Rosenblatt, "The perceptron: a probabilistic model for information storage and organization in the brain," Psychological Review, vol. 65, no. 6, p. 386, 1958.

[28] J. L. McClelland, D. E. Rumelhart, and P. R. Group, "Parallel distributed processing," Explorations in the Microstructure of Cognition, vol. 2, pp. 216-271, 1986.
[29] F. Rosenblatt, The Perceptron, a Perceiving and Recognizing Automaton Project Para, Cornell Aeronautical Laboratory, Buffalo, NY, USA, 1957.

[30] P. Werbos, "Beyond regression: new tools for prediction and analysis in the behavioral science," Thesis (Ph. D.), Harvard University, Cambridge, MA, USA, 1974.

[31] D. E. Rumelhart, G. E. Hinton, and R. J. Williams, "Learning representations by back-propagating errors," Nature, vol. 323, no. 6088, pp. 533-536, 1986.

[32] Y. Chen, J. Fan, Z. Deng et al., "PR-KELM: icing level prediction for transmission lines in smart grid," Future Generation Computer Systems, vol. 102, pp. 75-83, 2020. 